\title{
Quantitative T2 mapping of rats with chronic hepatitis
}

\author{
XIAOFENG WANG, YAN HU, XIN LU, YU CAI and JIAN SHU
}

Department of Radiology, The Affiliated Hospital of Southwest Medical University, Luzhou, Sichuan 646000, P.R. China

Received July 19, 2020; Accepted December 4, 2020

DOI: $10.3892 /$ etm. 2021.9656

\begin{abstract}
The aim of the study was to explore the diagnostic value of T2 mapping in an experimental rat model of chronic liver disease. Chronic hepatitis was induced in Sprague-Dawley male rats $(n=88)$ by intraperitoneal and abdominal subcutaneous injection of carbon tetrachloride in olive oil. The normal control rats $(n=12)$ were similarly injected with the same dose of normal saline. All rats were randomly selected and subjected to T2-weighted/spectral adiabatic inversion recovery and multiple gradient- and spin-echo sequence. After scanning, rats were sacrificed immediately and livers removed for staining with hematoxylin and eosin, as well as Masson's trichrome, to determine the pathological stage of hepatic fibrosis, necroinflammatory activity and steatosis. The T2 values were measured and associated with histopathological findings. The T2 values were significantly associated with hepatic fibrosis $(\mathrm{P}<0.05)$, but not with hepatitis $(\mathrm{P}>0.05)$ or steatosis $(\mathrm{P}>0.05)$. By partial correlation analysis, a significant positive correlation was observed between the T2 values and stages of liver fibrosis $(r=0.820 ; \mathrm{P}<0.05)$. T2 values increased with progressive hepatic fibrosis. The differences between $\mathrm{T} 2$ values and stages of liver fibrosis were statistically significant. Statistically significant differences were observed between different stages of liver fibrosis $(\mathrm{P}<0.05)$, with an area under the curve value of 0.944 for predicting stage F1 or greater, 0.942 for stage F2 or greater, 0.958 for stage F3 or greater, and 0.948 for F4. Thus, the $\mathrm{T} 2$ value is one of the quantitative indices of imaging and accurately reflects the stages of liver fibrosis.
\end{abstract}

\section{Introduction}

Chronic liver disease refers to chronic inflammation and fibrosis, representing major pathological changes induced by various etiologies lasting $>6$ months. Liver fibrosis is a wound-healing response generated against a wide range of underlying injuries. It can progress to liver cirrhosis, causing portal hypertension, hepatocellular carcinoma and liver failure. Liver fibrosis is a reversible process (1). If liver fibrosis

Correspondence to: Dr Jian Shu, Department of Radiology, The Affiliated Hospital of Southwest Medical University, 25 Taiping Street, Luzhou, Sichuan 646000, P.R. China

E-mail: shujiannc@163.com

Key words: magnetic resonance imaging, rat model, T2 value, chronic hepatitis is detected early, recovery is close to normal, but complete recovery from cirrhosis is not possible (2).

Early diagnosis of liver fibrosis is a critical step for early intervention, detection of disease course, and prognosis of various chronic liver diseases $(3,4)$. Diagnosis of chronic liver diseases is mainly based on patients' signs and symptoms, laboratory serological markers of liver function and histopathological examination. Currently, liver biopsy remains the gold standard for the assessment of liver fibrosis. However, biopsy is limited primarily by its inherent invasiveness and inconsistency due to blind puncture and sampling errors $(5,6)$.

Noninvasive diagnostic tests facilitate the assessment of the severity of chronic liver disease. However, relatively few diagnostic tests are noninvasive. In various chronic liver diseases, liver cell degeneration, necrosis, fibrosis and steatosis result in inhomogeneity of liver magnetic field and influence the quantized value of magnetic resonance imaging (MRI). Currently, several studies are focused on the role of quantitative MR in the staging of chronic hepatitis, including T1 mapping $(5,7)$ and multiecho gradient recalled echo T2 star weighted imaging (T2*WI) (7), diffusion-weighted imaging $(8,9)$, magnetic resonance elastography $(10,11)$, magnetic resonance spectroscopy (12), and dynamic contrast-enhanced MRI with gadolinium ethoxybenzyl diethylenetriamine penta-acetic acid $(13,14)$.

The $\mathrm{T} 2$ value can be used to evaluate the histological staging of liver fibrosis in animal models and humans with chronic liver disease $(15,16)$, but cannot distinguish between inflammation and fibrosis (17). The T2 value increases with increasing tissue water content and is decreased with increasing liver iron content, while the $\mathrm{R} 2$ value is increased with increasing liver iron content (T2=1/R2) (18-20). The R2 value can be used to quantify liver iron overload noninvasively and effectively, and liver fibrosis and iron concentration affect the measurement of $\mathrm{R} 2$ relaxation rate (21). T2 mapping not only reveals the changes in liver morphology but also partially reflects the liver metabolism biochemically. Therefore, the purpose of the present study was to explore the diagnostic role of T2 mapping in hepatic fibrosis $(\mathrm{F})$, necroinflammatory activity (A), and steatosis $(\mathrm{S})$ in a rat model of chronic hepatitis by injecting carbon tetrachloride $\left(\mathrm{CCl}_{4}\right)$, based on pathological findings as the reference standard.

\section{Materials and methods}

Liver fibrosis model. One-hundred male adult Sprague-Dawley rats $(210 \pm 5.2 \mathrm{~g} ; \mathrm{n}=100$; age, $6-8$ weeks) were randomly divided into two groups: A chronic hepatitis model group $(n=88)$ and 
a normal control group $(n=12)$. Before the experiment started, the rats were raised for a week. Forty-four rats were injected intraperitoneally with a mixture of $\mathrm{CCl}_{4}$ in olive oil (2:3) at a dose of $0.3 \mathrm{ml} / 100 \mathrm{~g}$ for $5-12$ weeks, twice a week. Another 44 rats were treated via subcutaneous injection abdominally to induce chronic hepatitis. The model simulated the evolution mechanism of chronic viral hepatitis. Six rats were induced by intraperitoneal injection with the same dose of normal saline (NS), and another six rats were induced via abdominal subcutaneous injection. All animals were fed under the same conditions of the facility at a temperature of $27 \pm 1^{\circ} \mathrm{C}$, at a humidity of $40-60 \%$, with free access to food and water.

MRI experiments. Imaging was performed using a $3.0 \mathrm{~T}$ MRI scanner (Achieva 3.0 T, Philips Healthcare) with an eight-channel knee joint coil. The scanning range extended from the top of the diaphragm to the lower edge of the liver. After 5-12 weeks post-injection, three to six rats injected abdominally to develop models of chronic hepatitis, four to six rat models of chronic hepatitis injected subcutaneously and two normal rats from the control group were randomly selected for T2-weighted/spectral adiabatic inversion recovery (T2WI-SPAIR) and multiple gradient- and spin-echo (M-GRASE) scan every week. Before scanning, rats were anesthetized by intraperitoneal injection of $1 \%$ pentobarbital $(50 \mathrm{mg} / \mathrm{kg})$. When the anesthetic effect was poor, a small amount of additional dose was appropriate. The parameters of T2WI-SPAIR included echo time (TE), $70 \mathrm{msec}$; repetition time (TR), $544 \mathrm{~ms}$; field of view (FOV), 90x67 mm²; slice thickness, $2.5 \mathrm{~mm}$; slice gap, $0.3 \mathrm{~mm}$; number of excitations (NEXs), 1; flip angle, 90 degrees; and scan time, 2'10.6". The parameters of M-GRASE included TEs, 19, 38, 57, 76 and $95 \mathrm{msec}$; TR, $1660 \mathrm{msec}$; FOV, 120x120 mm²; slice thickness, $2.5 \mathrm{~mm}$; slice gap, $0.6 \mathrm{~mm}$; NEX, 1; flip angle, 90 degrees; and scan time, 2' $26^{\prime \prime}$. The T2 values were measured on the T2 map obtained from images of different TE and reconstructed automatically.

Histology studies. After scanning, the aforementioned anesthetized rats were sacrificed immediately by cervical dislocation and the livers removed were stained with H\&E and Masson's trichrome. The specimens were fixed in $10 \%$ formalin solution for $18-24 \mathrm{~h}$ under the room temperature. After washing, dehydrating and decalcifying, the specimens were embedded in paraffin wax and then sectioned at the thickness of $5 \mu \mathrm{m}$. H\&E was performed following the instructions of the Hematoxylin and Eosin Staining kit (Beyotime Institute of Biotechnology). Masson trichromatic staining was carried out according to the instructions of the Masson trichromatic Staining kit (Beijing Solarbio Science \& Technology Co., Ltd.). All dyed slices were assessed by two pathologists, who worked for more than 10 years in a double-blind method. The stages of hepatic fibrosis (F) and necroinflammatory activity (A) were assessed pathologically according to the METAVIR scoring system (22). F was staged on a scale of 0 to 4: F0, no fibrosis; $\mathrm{F} 1$, portal fibrosis without septa; F2, portal fibrosis with rare septa; F3, numerous septa without cirrhosis; and F4, cirrhosis. A was determined according to the severity of portal, periportal and lobular inflammation and was classified as follows: A0, no activity; A1, mild activity; and A3, severe activity. Hepatic steatosis (S) was measured by determining the percentage of fatty infiltration, which was graded as S0 (0-5\%), S1 (6-30\%), S2 (31-50\%), S3 (51-75\%) and S4 (>75\%). If the diagnoses of the two doctors were inconsistent, the final pathological result was obtained based on subsequent discussion. The normal control rats with hepatitis, liver fibrosis or fatty liver were not included in the statistical analysis.

Image analysis. All images were processed in the Philips Extended MR WorkSpace 2.6.3.4. The images with abnormal signal and artifact interference on the liver were deleted. The $\mathrm{T} 2$ values were measured by an experienced radiologist in cases lacking pathological results. Three ROIs (region of interest) of $10-\mathrm{mm}^{2}$ area were placed on $\mathrm{T} 2$ map that showed the maximum liver area (Fig. 1).

Data analysis. Multiple linear regression analysis (the enter method) was performed to determine the T2 values that associated with stages of hepatic fibrosis, necroinflammatory activity and steatosis. The correlation between $\mathrm{T} 2$ value and hepatic fibrosis and steatosis were analyzed independently via partial correlation analysis to determine the correlation coefficient ( $r$ value). Based on the results of multiple linear regression analysis, the differences in T2 value between the stages of liver fibrosis were tested by one-way analysis of variance, and the differences between the groups were analyzed using the Tukey's test. The accuracy of the T2 values for the assessment of fibrosis stage was evaluated using the receiver operating characteristic (ROC) curves. All statistical analyses were performed using SPSS software (version 17.0; SPSS, Inc.). $\mathrm{P}<0.05$ was considered to indicate a statistically significant difference.

\section{Results}

Pathology results. Twenty-five rats from the chronic hepatitis group died of systemic failure caused by acute hepatic necrosis, abdominal infection or intestinal obstruction. The mortality rate was $28.41 \%$. One rat was not included in the statistical analysis because of hepatitis in the normal control group. The F0A0S0 results were available for 11 normal control rats. The pathology results of 63 rats with chronic hepatitis were as follows: F1 $(n=19), F 2(n=16), F 3(n=13), F 4$ $(n=15), A 1(n=31), A 2(n=22), A 3(n=10) ; S 1(n=12), S 2(n=12)$, S3 $(n=19)$, and S4 $(n=20)$ (Table I). The pathology results and T2 maps at different stages of hepatic fibrosis are shown in Figs. 2-4.

Statistical analysis. Based on the results of multiple linear regression analysis (Table II), the T2 value was closely associated with $\mathrm{F}(\mathrm{P}=0.000)$, but not with $\mathrm{A}(\mathrm{P}=0.052)$ or $\mathrm{S}(\mathrm{P}=0.409)$. According to the partial correlation analysis, a significant positive correlation was detected between the $\mathrm{T} 2$ value and the staging of liver fibrosis $(r=0.820 ; \mathrm{P}<0.05$; Table II). As shown in Table III, the average T2 value of different stages of liver fibrosis increased with progressive hepatic fibrosis. The difference between T2 value and the stages of liver fibrosis were statistically significant $(F=55.61 ; \mathrm{P}=0.000)$. There were statistical differences between each stage of T2 value as follows: $\mathrm{F} 0$ and $\mathrm{F} 1$ $(\mathrm{P}=0.029), \mathrm{F} 0$ and F2 (P=0.000), F0 and F3 $(\mathrm{P}=0.000), \mathrm{F} 0$ and 
Table I. Distribution of T2 values in liver pathogenesis $($ mean $\pm \mathrm{SD})$.

\begin{tabular}{ll}
\hline Pathologic staging (n) & T2 value, ms \\
\hline A0F0S0 (11) & $34.68 \pm 1.59$ \\
A1F1S1 (5) & $34.98 \pm 2.76$ \\
A1F1S2 (2) & $39.13 \pm 6.43$ \\
A1F1S4 (3) & $38.07 \pm 4.96$ \\
A1F2S1 (2) & $44.96 \pm 2.78$ \\
A1F2S2 (4) & $39.38 \pm 2.08$ \\
A1F2S3 (3) & $44.45 \pm 3.99$ \\
A1F2S4 (4) & $44.26 \pm 4.31$ \\
A1F3S3 (2) & $44.91 \pm 2.65$ \\
A1F3S4 (2) & $48.51 \pm 1.38$ \\
A1F4S2 (2) & $50.38 \pm 1.66$ \\
A1F4S3 (1) & $47.43 \pm 0$ \\
A1F4S4 (1) & $49.77 \pm 0$ \\
A2F1S3 (2) & $41.34 \pm 6.17$ \\
A2F1S4 (3) & $39.82 \pm 1.26$ \\
A2F2S1 (1) & $40.47 \pm 0$ \\
A2F2S3 (1) & $45.15 \pm 0$ \\
A2F3S1 (1) & $47.45 \pm 0$ \\
A2F3S2 (1) & $45.03 \pm 0$ \\
A2F3S3 (1) & $40.23 \pm 0$ \\
A2F3S4 (2) & $49.80 \pm 4.67$ \\
A2F4S1 (2) & $51.00 \pm 3.92$ \\
A2F4S2 (3) & $52.29 \pm 3.13$ \\
A2F4S3 (4) & $52.41 \pm 4.09$ \\
A2F4S4 (1) & $55.55 \pm 0$ \\
A3F1S3 (2) & $41.27 \pm 0.29$ \\
A3F1S4 (2) & $40.47 \pm 0.14$ \\
A3F2S4 (1) & $45.13 \pm 0$ \\
A3F3S1 (1) & $49.40 \pm 0$ \\
A3F3S3 (2) & $51.04 \pm 3.73$ \\
A3F3S4 (1) & \\
A3F4S3 (1) & \\
\hline & \\
&
\end{tabular}

F4 $(\mathrm{P}=0.000), \mathrm{F} 1$ and $\mathrm{F} 2(\mathrm{P}=0.002), \mathrm{F} 1$ and $\mathrm{F} 3(\mathrm{P}=0.000), \mathrm{F} 1$ and F4 $(\mathrm{P}=0.000), \mathrm{F} 2$ and $\mathrm{F} 3(\mathrm{P}=0.004), \mathrm{F} 2$ and $\mathrm{F} 4(\mathrm{P}=0.000)$, $\mathrm{F} 3$ and $\mathrm{F} 4(\mathrm{P}=0.020)$. Fig. 5 shows the ROC curves based on different thresholds of fibrosis stages. The area under the ROC curve (AUC), optimal cutoff values, as well as the corresponding sensitivity and specificity, are presented in Table IV.

\section{Discussion}

The present study analyzed the association between the T2 value of hepatic parenchyma and the histological grade of liver fibrosis, steatosis and hepatitis activity in a toxic model of chronic hepatitis, in which rats were injected with a suspension of $\mathrm{CCl}_{4}$. The results show that the $\mathrm{T} 2$ value was associated with hepatic fibrosis, but not with steatosis or hepatitis. The $\mathrm{T} 2$ value increases with advanced fibrosis. The AUC obtained using T2 values was 0.944 for the prediction of stage F1 or
Table II. Results of regression analysis correlating T2 values and pathology.

\begin{tabular}{lccc}
\hline & \multicolumn{3}{c}{ T2 value } \\
\cline { 2 - 4 } Pathologic staging & $B$ & P-value & $r$ \\
\hline F & 3.904 & 0.000 & 0.820 \\
A & 1.061 & 0.052 & 0.230 \\
S & 0.270 & 0.409 & 0.099 \\
\hline
\end{tabular}

$B$ value, unstandardized coefficient. $r$ value, partial correlation coefficient. F, hepatic fibrosis; necroinflammatory activity; S, steatosis.

Table III. Distribution of the T2 value in the different stages of liver fibrosis.

\begin{tabular}{lc} 
Stages of liver fibrosis $(n)$ & T2 value, mean \pm SD \\
\hline F0 $(n=11)$ & $34.68 \pm 1.58$ \\
F1 $(n=19)$ & $38.58 \pm 3.87$ \\
F2 $(n=16)$ & $43.04 \pm 3.62$ \\
F3 $(n=13)$ & $47.77 \pm 3.72$ \\
F4 $(n=15)$ & $51.85 \pm 3.26$
\end{tabular}

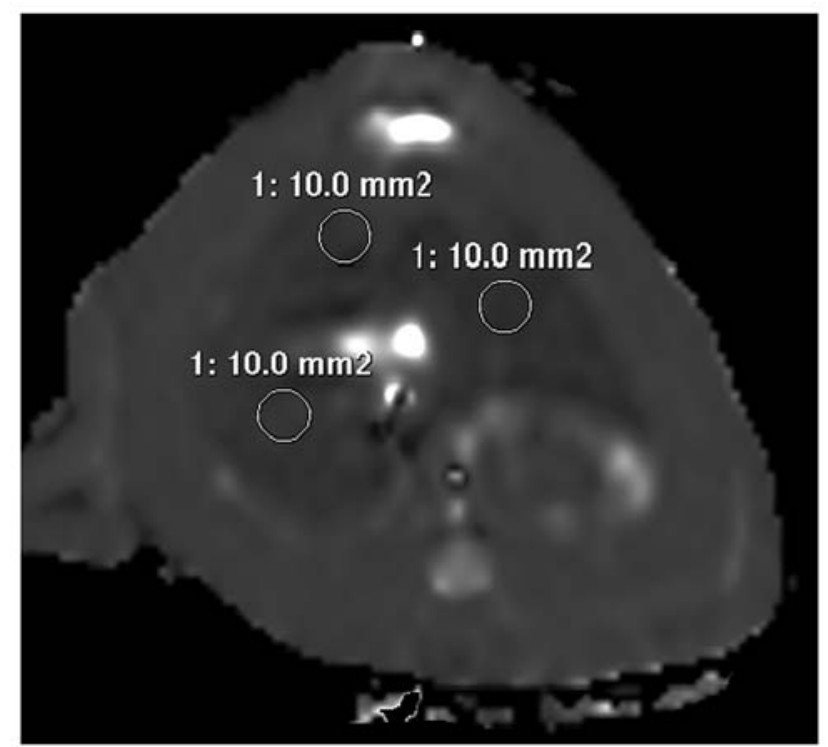

Figure 1. Measurement ROIs shown in the T2 map. Three ROIs are located on the image with the largest slice of liver area. At least one ROI with $10-\mathrm{mm}^{2}$ area is located on the left or right lobes of the liver, avoiding intrahepatic bile ducts, blood vessels and artifacts, and at least $3 \mathrm{~mm}$ from the edge of the liver. ROI, region of interest.

greater, 0.942 for stage F2 or greater, 0.958 for stage F3 or greater, and 0.948 for F4.

Inflammatory necrosis and fibrosis of liver tissue, the most fundamental pathological basis of chronic hepatitis, are characterized by liver cell degeneration, necrosis and apoptosis to varying degrees. With the infiltration of inflammatory cells in the portal area, the activation of hepatic stellate cells, 
Table IV. Diagnostic performance of T2 value in the staging of liver fibrosis.

\begin{tabular}{lcccc}
\hline Statistic & F0 vs. F1-4 & F0,1 vs. F2-4 & F0-2 vs. F3,4 & F0-3 vs. F4 \\
\hline AUC & 0.944 & 0.942 & 0.956 & 0.948 \\
P-value & 0.000 & 0.000 & 0.000 & 0.000 \\
Cut-off value & 37.315 & 42.55 & 46.10 & 46.83 \\
Sensitivity, \% & 88.89 & 81.82 & 89.29 & 100.00 \\
Specificity, \% & 100.00 & 93.33 & 91.30 & 81.36 \\
Positive predictive value, \% & 100.00 & 94.74 & 86.21 & 57.69 \\
Negative predictive value, \% & 61.10 & 77.78 & 93.30 & 100.00
\end{tabular}

AUC, area under the curve; F, hepatic fibrosis.

F0

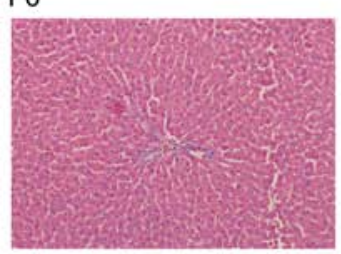

F1

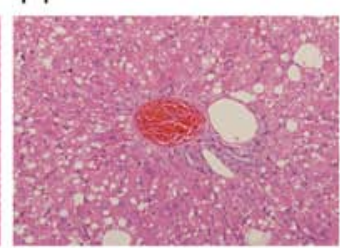

F2

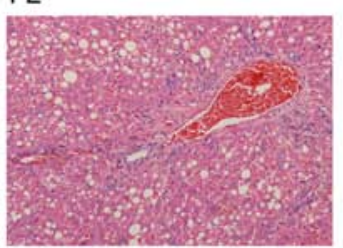

F3

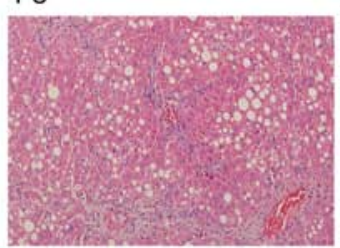

F4

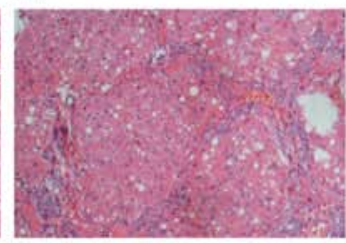

Figure 2. H\&E staining of liver in rats with chronic hepatitis F0 (magnification, x100) and F1-4 (magnification, x200), with different grades of inflammation and steatosis. F0, normal liver tissue; F1, portal fibrosis without septa; F2, portal fibrosis with rare septa; F3, numerous septa without cirrhosis; and F4, cirrhosis. F, hepatic fibrosis.

FO

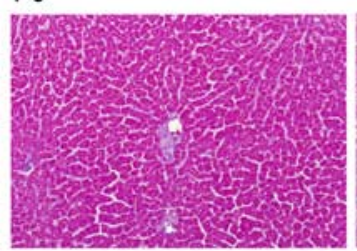

F1

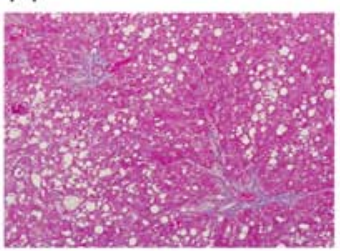

F2

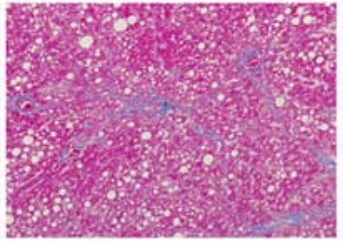

F3

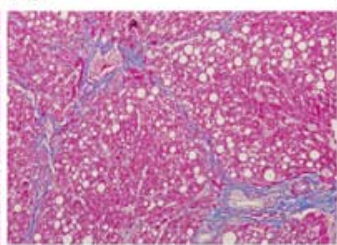

F4

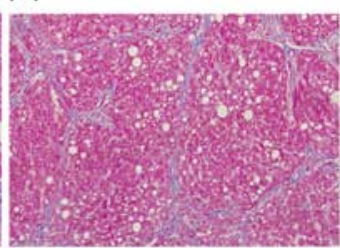

Figure 3. Masson's trichrome staining of liver in rats with chronic hepatitis F0 (magnification, x100) and F1-4 (magnification, x200), with different grades of inflammation and steatosis. The whole blue stained area is hepatic fibrosis. F0, normal liver tissue; F1, portal fibrosis without septa; F2, portal fibrosis with rare septa; F3, numerous septa without cirrhosis; and F4, cirrhosis. F, hepatic fibrosis.

F0

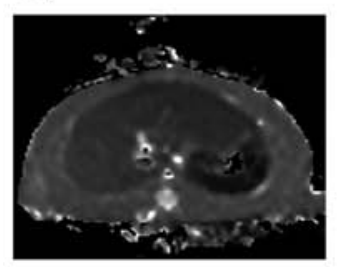

F1

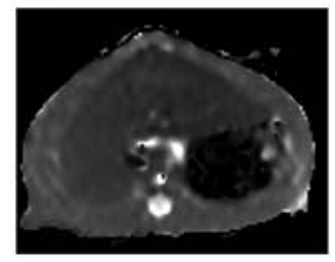

F2

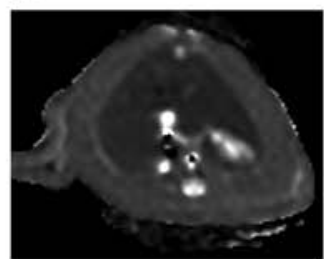

F3

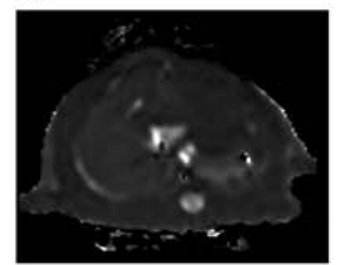

F4

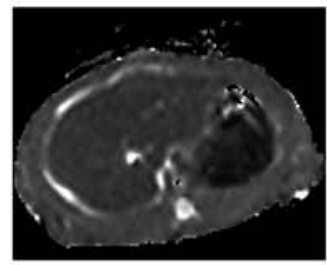

Figure 4. T2 maps of liver fibrosis (F0-4). F0, normal liver tissue; F1, portal fibrosis without septa; F2, portal fibrosis with rare septa; F3, numerous septa without cirrhosis; and F4, cirrhosis. F, hepatic fibrosis.

excessive extracellular matrix (ECM) hyperplasia and deposition result in fibrous scar formation eventually (23-25). A close topographical association exists between inflamed areas of the liver and areas that develop fibrosis. The presence of these inflammatory cells may increase the T2 value (16). In addition, hepatocyte necrosis and rupture result in a large and abnormal accumulation of ferritin. Iron deposition further damages the liver by inducing oxidative stress in the liver cells (14). All these processes alter the T2 value. In our study group, the hepatic T2 value was not associated with hepatitis or steatosis.

In recent years, MRI has been increasingly used for the diagnosis and staging of chronic liver diseases. A few studies reported T2 mapping techniques to grade liver fibrosis. Zhang et al (26) reported that T2 value correlated positively with stages of liver fibrosis, and increased T2 value was observed in severe liver fibrosis. Spearman's correlation test showed that the mean $\mathrm{T} 2$ value correlated positively 


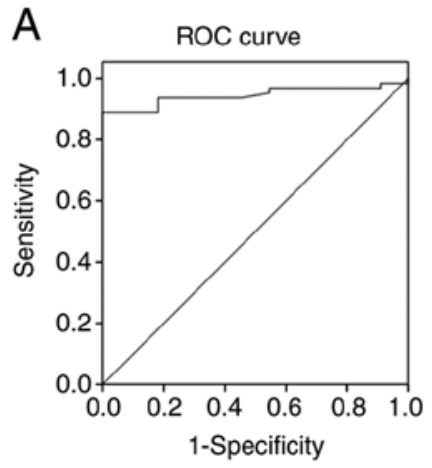

Diagonal segments are produced by ties.
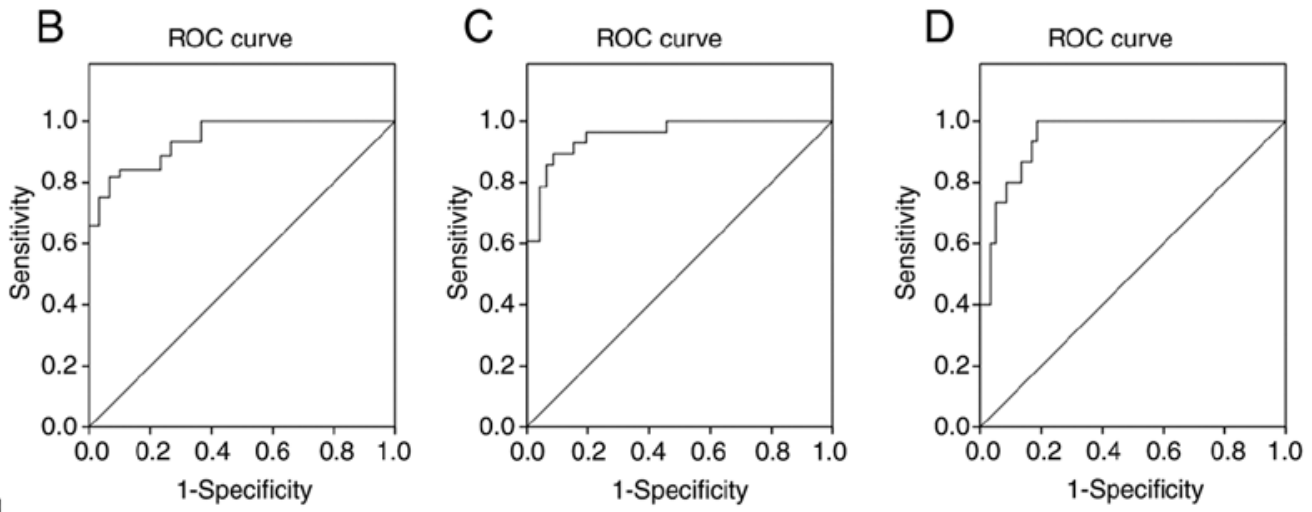

Figure 5. ROC curves for T2 values for the determination of fibrotic stage thresholds. (A) F0 vs. F1-4 (AUC, 0.944); (B) F0-1 vs. F2-4 (AUC, 0.942); (C) F0-2 vs. F3-4 (AUC, 0.958); (D) F0-3 vs. F4 (AUC, 0.948). ROC, receiver operating characteristics; F, hepatic fibrosis; AUC, area under curve.

$(\mathrm{r}=0.73 ; \mathrm{P}<0.001)$ with fibrosis stages. Guimaraes et al $(16)$ demonstrated an increased $\mathrm{T} 2$ value with increasing stage of liver fibrosis in a diethylnitrosamine rat model of liver fibrosis, without evidence of hemochromatosis or fatty infiltration within the liver. The T2 relaxation time can potentially separate patients with mild disease from patients with severe liver fibrosis, based on a statistically significant difference between degrees of mild vs. severe fibrosis $(\mathrm{P}=0.03)$. Luetkens et al (27) used two different models of chronic liver disease in rats, including the cholestatic model of liver fibrosis, induced by bile duct ligation, and a toxic model of liver fibrosis, in which rats were exposed to periodic $\mathrm{CCl}_{4}$ inhalation. The results demonstrated that the quantitative values of hepatic $\mathrm{T} 2$ can be used to differentiate between the stages of liver fibrosis based on histology.

The present study yielded similar results. The T2 value of hepatic parenchyma was closely related to hepatic fibrosis $(\mathrm{P}<0.05)$, but not to hepatitis or steatosis, and the difference in $\mathrm{T} 2$ value was statistically significant between the different stages of hepatic fibrosis. However, it is inconsistent with the results of Chow et al (15), who reported that the degree of fibrosis and inflammation in fibrotic livers remained relatively stable after week 4 and T2 value showed a higher sensitivity for the detection of early liver fibrosis rather than each stage of hepatic fibrosis. It may be attributed to different rat species and the effect of $\mathrm{CCL}_{4}$ doses on the degree of liver damage. The studies $(15,26,27)$ did not indicate the effects of liver inflammation on the T2 value. However, as mentioned earlier, inflammatory activity and steatosis affect the T2 value. Therefore, a comprehensive analysis of factors that affect the T2 value in patients with liver disease is needed.

Limitations. The study limitations are as follows: In this study, a single-animal model was used, and liver fibrosis was not analyzed using multiple modeling methods. The maximum image level without artifact was not rejected. Any artifacts associated with smaller images may be attributed to the low weight of the rats, respiratory movement, heartbeat, gastrointestinal peristalsis and intestinal gas. Hepatic iron deposition in liver fibers affects the T2 value. However, the classification and measurements were not corrected for hepatic iron overload in the present study. The association between liver iron overload and liver fibrosis was also not analyzed, nor the effect of liver iron overload on liver T2 value. Further studies are needed to address these limitations.

Conclusions. The experiments showed that the T2 value was mainly affected by liver fibrosis, but was not associated with hepatitis or fatty liver. It was positively correlated with the T2 value and staging of liver fibrosis. The T2 value is one of the indices of quantitative image analysis and reflects the stage of liver fibrosis.

\section{Acknowledgements}

Not applicable.

\section{Funding}

This research was funded by the Science and Technology program of Luzhou (grant no. 2015LZYD-S04).

\section{Availability of data and materials}

The datasets used and/or analyzed during the current study are available from the corresponding author on reasonable request.

\section{Authors' contributions}

JS designed the experiments. XW and XL collected samples and performed experiments. XW, YH, XL, YC and JS collected and assembled data. XW, YH and JS analyzed and interpreted the data. XW, YH and JS were involved in drafting the manuscript and revising it critically for important intellectual content. All the authors read and approved the final manuscript. JS and XW confirm the authenticity of all the raw data.

\section{Ethics approval and consent to participate}

Experiments were carried out in accordance with the International Guidelines for Animal Studies regarding the care and use of animals for experimental purposes. All animal experiments followed a protocol approved by the local Institutional Animal Ethics Committee: The Southwest Medical University 
(Luzhou, China) and the Laboratory animal production licenses were SCXK (Chuan) 2013-17, SCXK (Chuan) 2013-181 and SCXK (Chuan) 2013-065.

\section{Patient consent for publication}

Not applicable.

\section{Competing interests}

The authors declare that they have no competing interests.

\section{References}

1. Fowell AJ and Iredale JP: Emerging therapies for liver fibrosis. Dig Dis 24: 174-183, 2006.

2. Issa R, Zhou X, Constandinou CM, Fallowfield J, MillwardSadler H, Gaca MD, Sands E, Suliman I, Trim N, Knorr A, et al: Spontaneous recovery from micronodular cirrhosis: Evidence for incomplete resolution associated with matrix cross-linking. Gastroenterology 126: 1795-1808, 2004.

3. Venkatesh SK, Yin M and Ehman RL: Magnetic resonance elastography of liver: Technique, analysis, and clinical applications. J Magn Reson Imaging 37: 544-555, 2013.

4. Li BB, Li DL, Chen C, Liu BH, Xia CY, Wu HJ, Wu CQ, Ji GQ, Liu S, Ni W, et al: Potentials of the elevated circulating miR-185 level as a biomarker for early diagnosis of HBV-related liver fibrosis. Sci Rep 6: 34157, 2016.

5. Manning DS and Afdhal NH: Diagnosis and quantitation of fibrosis. Gastroenterology 134: 1670-1681, 2008.

6. Sporea I, Popescu A and Sirli R: Why, who and how should perform liver biopsy in chronic liver diseases. World J Gastroenterol 14: 3396-3402, 2008.

7. Banerjee R, Pavlides M, Tunnicliffe EM, Piechnik SK, Sarania N, Philips R, Collier JD, Booth JC, Schneider JE, Wang LM, et al: Multiparametric magnetic resonance for the non-invasive diagnosis of liver disease. J Hepatol 60: 69-77, 2014.

8. Zhou IY, Gao DS, Chow AM, Fan S, Cheung MM, Ling C, Liu X, Cao P, Guo H, Man K and Wu EX: Effect of diffusion time on liver DWI: An experimental study of normal and fibrotic livers. Magn Reson Med 72: 1389-1396, 2014.

9. Hu XR, Cui XN, Hu QT and Chen J: Value of MR diffusion imaging in hepatic fibrosis and its correlations with serum indices. World J Gastroenterol 20: 7964-7970, 2014.

10. Reiter R, Wetzel M, Hamesch K, Strnad P, Asbach P, Haas M, Siegmund B, Trautwein C, Hamm B, Klatt D, et al: Comparison of non-invasive assessment of liver fibrosis in patients with alpha1-antitrypsin deficiency using magnetic resonance elastography (MRE), acoustic radiation force impulse (ARFI) Quantification, and 2D-shear wave elastography (2D-SWE). PLoS One 13: e0196486, 2018.

11. Kim YS, Jang YN and Song JS: Comparison of gradient-recalled echo and spin-echo echo-planar imaging MR elastography in staging liver fibrosis: A meta-analysis. Eur Radiol 28: 1709-1718, 2018.
12. Wang XM, Zhang XJ and Ma L: Diagnostic performance of magnetic resonance technology in detecting steatosis or fibrosis in patients with nonalcoholic fatty liver disease: A meta-analysis. Medicine (Baltimore) 97: e10605, 2018.

13. Chen BB, Hsu CY, Yu CW, Wei SY, Kao JH, Lee HS and Shih TT: Dynamic contrast-enhanced magnetic resonance imaging with Gd-EOB-DTPA for the evaluation of liver fibrosis in chronic hepatitis patients. Eur Radiol 22: 171-180, 2012.

14. Zhang W, Kong X, Wang ZJ, Luo S, Huang W and Zhang LJ: Dynamic contrast-enhanced magnetic resonance imaging with Gd-EOB-DTPA for the evaluation of liver fibrosis induced by carbon tetrachloride in rats. PLoS One 10: e0129621, 2015.

15. Chow AM, Gao DS, Fan SJ, Qiao Z, Lee FY, Yang J, Man K and Wu EX: Measurement of liver T1 and T2 relaxation times in an experimental mouse model of liver fibrosis. J Magn Reson Imaging 36: 152-158, 2012.

16. Guimaraes AR, Siqueira L, Uppal R, Alford J, Fuchs BC, Yamada S, Tanabe K, Chung RT, Lauwers G, Chew ML, et al: T2 relaxation time is related to liver fibrosis severity. Quant Imaging Med Surg 6: 103-114, 2016.

17. Jacob RE, Amidan BG, Soelberg J and Minard KR: In vivo MRI of altered proton signal intensity and $\mathrm{T} 2$ relaxation in a bleomycin model of pulmonary inflammation and fibrosis. J Magn Reson Imaging 31: 1091-1099, 2010

18. Giri S, Chung YC, Merchant A, Mihai G, Rajagopalan S, Raman SV and Simonetti OP: T2 quantification for improved detection of myocardial edema. J Cardiovasc Magn Reson 11: $56,2009$.

19. Naßenstein K, Nensa F, Schlosser T, Bruder O, Umutlu L, Lauenstein T, Maderwald S and Ladd ME: Cardiac MRI: T2-mapping versus T2-weighted dark-blood TSE imaging for myocardial edema visualization in acute myocardial infarction. Rofo 186: 166-172, 2014.

20. St Pierre TG, Clark PR and Chua-Anusorn W: Measurement and mapping of liver iron concentrations using magnetic resonance imaging. Ann N Y Acad Sci 1054: 379-385, 2005.

21. Alexopoulou E, Stripeli F, Baras P, Seimenis I, Kattamis A, Ladis V, Efstathopoulos E, Brountzos EN, Kelekis AD and Kelekis NL: R2 relaxometry with MRI for the quantification of tissue iron overload in beta-thalassemic patients. J Magn Reson Imaging 23: 163-170, 2006.

22. Bedossa P and Poynard T: An algorithm for the grading of activity in chronic hepatitis C. The METAVIR Cooperative Study Group. Hepatology 24: 289-293, 1996.

23. Kisseleva T and Brenner DA: Mechanisms of fibrogenesis. Exp Biol Med (Maywood) 233: 109-122, 2008.

24. Yanguas SC, Cogliati B, Willebrords J, Maes M, Colle I, van den Bossche B, de Oliveira CPMS, Andraus W, Alves VAF, Leclercq I and Vinken M: Experimental models of liver fibrosis. Arch Toxicol 90: 1025-1048, 2016.

25. Aydın MM and Akçalı KC: Liver fibrosis. Turk J Gastroenterol 29: 14-21, 2018.

26. Zhang H, Yang Q, Yu T, Chen X, Huang J, Tan C, Liang B and Guo H: Comparison of T2, T1rho, and diffusion metrics in assessment of liver fibrosis in rats. J Magn Reson Imaging 45: 741-750, 2017.

This work is licensed under a Creative Commons Attribution-NonCommercial-NoDerivatives 4.0 International (CC BY-NC-ND 4.0) License. 